\title{
Chondromas and multiple enchondromatosis
}

\author{
P. Lascombes ${ }^{1}$, L. Mainard-Simard ${ }^{2}$
}

${ }^{1}$ University of Nancy, France; Geneva University Hospitals, Geneva, Switzerland

${ }^{2}$ Paediatric Radiology, Hôpital d'Enfants, CHU de Nancy, Vandoeuvre les Nancy, France

Introduction The chondromas are a cartilaginous proliferation of mature appearance and moderate size, reason why these tumors are regarded more like hamartomas than real benign tumor. Chondromas represent 10 to $12 \%$ of benign bone tumors. Any bone of an enchondral ossification may be involved. Several bones can be involved, and the disease is called "chondromatosis". In the review we describe clinical and radiological findings of this pathology as well as indications for reconstructive surgery. Material and methods The review is dedicated to isolated chondromas, periosteal and extraskeletal chondromas, chondromatosis. Results The aspects of epidemiology, clinical presentation, radiology, MRI, prognosis, indications and methods of surgical treatment have been described in the article for each types of chondroma and enchondromatosis. Conclusion Chondromas are benign bone tumors which may be responsible of pathologic fractures. Their surgical treatment consists in curettage and bone grafting or bone-cement filling with or without osteosynthesis. Multiple enchondromatosis should be considered as an osteochondrodysplasia. Its treatment is not the treatment of the multiple chondromas themselves, but of the bone deformities and length discrepancy induced by the disorder. The transformation of some tumors in chondrosarcomas in adolescence or adulthood needs a strict follow up of these patients.

Keywords: chondromas, diagnostics, surgical treatment

\section{INTRODUCTION}

The chondromas (condrion) are a cartilaginous proliferation of mature appearance and moderate size, the reason why these tumors are regarded more like hamartomas than real benign tumours [1, 2]. They mainly develop within or alongside the bone. They are also called "enchondromas". Some other chondromas are periosteal chondromas as they develop close to the bone. Chondromas represent 10 to $12 \%$ of benign bone tumours. Any bone of an enchondral ossification may be involved, usually only one bone. In some circumstances, several bones are involved, and the disease is called "chondromatosis".

\section{RESULTS AND DISCUSSION}

\section{Isolated chondroma}

\subsection{Epidemiology}

Males and females are equally affected. The age range at diagnosis is between 10 and 40 years old. Any bone can be involved, from the most to less frequent:

- Long bones of hand and foot (till $50 \%$ )

- Femur, humerus, tibia

- Other long bones in their metaphyseal area

- Short bones of hand and foot

- Vertebra, skull basis

\subsection{Diagnosis}

The diagnosis is evoked in three circumstances: 1 chondromas are often discovered on an X-ray required for another reason (Fig. 1); 2 - in a context of a tumour

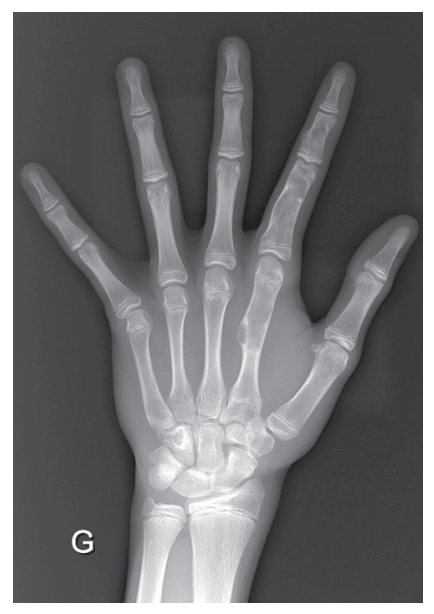
multiple chondromas of the $4^{\text {th }}$ metacarpal bone and finger
Fig. 1 Asymptomatic

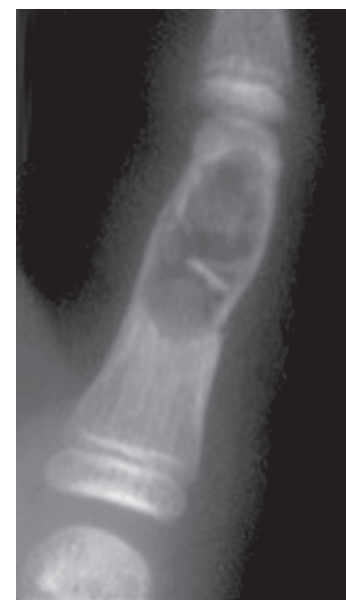

Fig. 2 Pathologic fracture due to chondroma of the first phalanx with an increase of the volume of the bone, associated with pain. This is frequent in fingers; 3 - in case of a pathologic fracture after minor trauma (Fig. 2).

\subsection{X-rays}

The typical chondroma is a localised osteolysis, geographic and polycyclic, in the centrum of the bone and with perfect limits. The bone may be "ballooned". Inside the lytic area, some annular calcifications enclose small cartilaginous nodules. The appearance of a cloudy "popcorn" aspect is highly suggestive of chondroma (Fig. 3, 4). The limits of the tumour are sometimes sclerotic. The cortex can be very thin, but never broken except in a case of a pathological fracture. Finally, there is neither periosteum reaction nor invasion of the soft tissues.
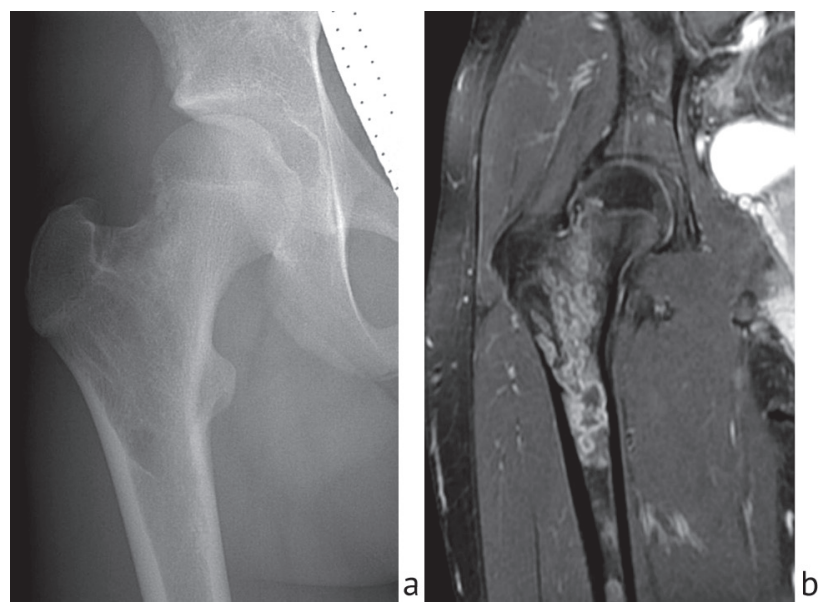

Fig. 3 Chondromas of the proximal femur in a 12-year old girl. X-ray (a), MRI with gadolinium (b)

Lascombes P., Mainard-Simard L. Chondromas and multiple enchondromatosis. Genij Ortopedii, 2021, vol. 27, no 4, pp. 441-445. DOI 10.18019/1028-4427-2021-27-4-441-445 


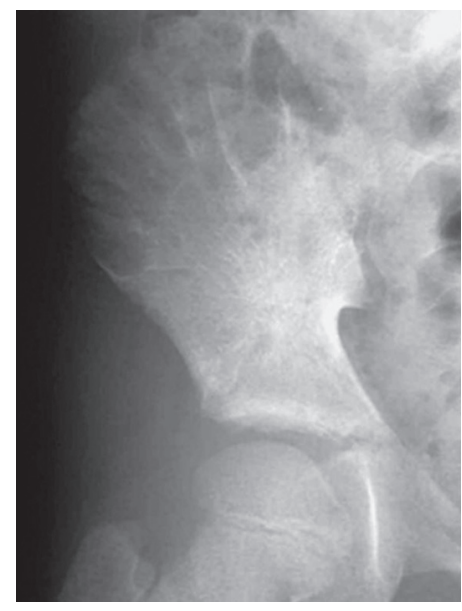

Fig. 4 Chondroma of the iliac crest. Observe the images of "candle casting"

\subsection{MRI}

The signal of the tumour is strictly comparable to the cartilage of the adjacent joints. There is a hypo signal on T1 sequences, a hyper signal on $\mathrm{T} 2$ sequences. Ossification spots are represented by black signals (Fig. 5). Globally, the mass is homogeneous. When the tumour is close to an epiphysis, some aspect like a "candle casting" may be observed.

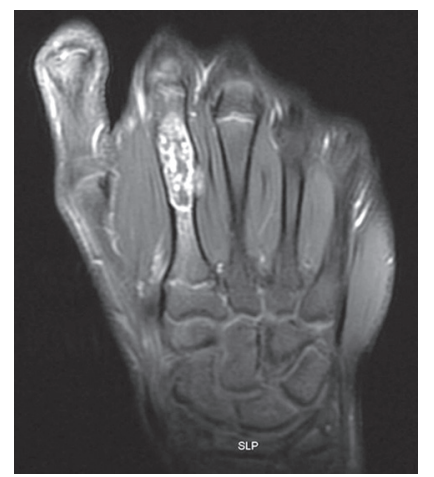

Fig. 5 MRI of a chondroma of the $2^{\text {nd }}$ metacarpal bone. Gadolinium. Notice the typical "popcorn" aspect

\subsection{Other types of chondromas}

\subsubsection{Periosteal chondroma}

The periosteal chondromas present with a development which is either subperiosteal or intraperiosteum. They frequently involve the cortex of the bone. They are observed in children and young adults. The X-rays show a defect of the cortex, mainly round or lobulated, with sclerotic margins [3]. On MRI, the tumour is typical with its cartilaginous mass and presence of numerous ossifications.

\subsubsection{Extraskeletal chondroma}

Some chondromas may develop into the soft tissues of the extremities, most often into the muscles and the tendon synovial sheaths (Fig. 6). The mean age is around the third and fourth decades.

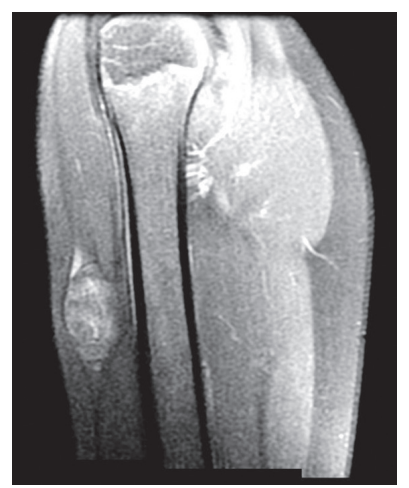

Fig. 6 Extraperiosteal chondroma of the anterior arm in a 10-year old boy, IRM

\subsection{Differential diagnosis}

In absence of intra tumoral calcifications, some lytic cartilaginous tumors may be confused with some bone cysts. The sclerotic limit of the chondromas may simulate some osteoblastomas. A chondromyxoid fibroma, frequently in the foot may also simulate some chondromas as well as a glomus tumor on the finger's extremities or invasion by nodules of villonodular synovitis. Most difficult is to differentiate some chondromas from low grade chondrosarcomas. In adults, the PET-CT can be used as an objective and quantitative method of differentiating between chondromas and chondrosarcomas [4].

\subsection{Pathology}

The tumor is characterized by nodules of mature hyaline cartilage. These nodules are separated by intervals of normal osteomedullary tissue. The chondrocytes cells are rare and small. Rarely, they may contain double nuclei. The main problem is to be sure to differentiate them from a low grade chondrosarcoma.

\subsection{Treatment}

Biopsy is not mandatory in typical forms like in fingers localisation. Clinical and radiologic observation is recommended in asymptomatic tumors. When clinical signs are present, like pain, or in case of a risk of pathological fracture, a complete curettage of the tumor lead to a usual healing of the lesion [5]. According to the localisation, it is most often preferable to add a bone graft after the curettage or to fill it up for some resorbable bone-cement [6]. Osteosythesis can also be required to prevent from a pathologic fracture.

The prognosis is good for extremities. However, chondromas of flat bones like scapula, sternum, and pelvis may transform some years later in chondrosarcomas.

\section{Chondromatosis}

\subsection{Description}

Chondromatosis is a disease which present with multiple chondromas. Several types are observed, the most frequent are:

- The enchondromatosis orOllier's dyschondroplasia

- The Maffucci's synsdrome with association of angiomas

- The Maffucci-Kaast syndrome with association of pigmentary anomalies

Enchondromatosis is known as the Ollier's disease and has been described par a French professor of orthopaedics in Lyon in 1898. Louis Xavier Édouard Léopold OLLIER (1830-1900) observed three patients with multiple tumors of the fingers [7]. These tumors looked like some exostosis and presented with a kind of bone mobility. With a per cutaneous needle, Ollier realized that the tissue had the same density than the cartilage. Some months later, he obtained X-rays which demonstrated the lytic bone tumors.

Enchondromatosis is not considered as an inherited condition, and cases are sporadic. The multiple chondromas may involve any bones. We can describe different forms:

- A minor type with some chondromas limited to the hands, or to one limb with minor symptoms

- A unilateral type with an involvement of one only limb or of one side of the body (Fig. 7).

- And a diffuse type with an involvement of the whole body. The asymmetry on the limbs between right and left sides is the rule. This condition is comparable with a kind of osteochondrodysplasica (Fig. 8). 


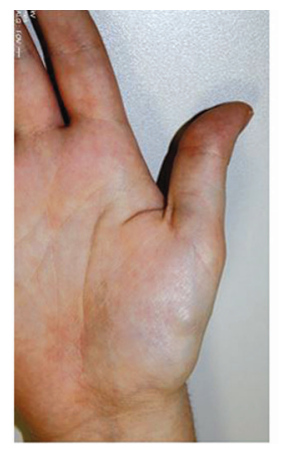

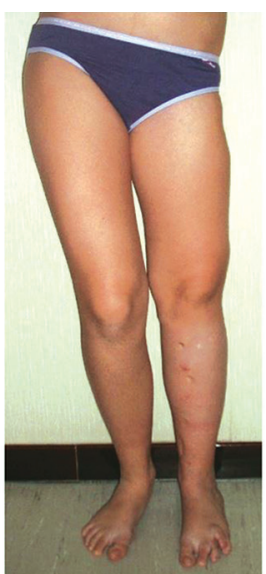

b

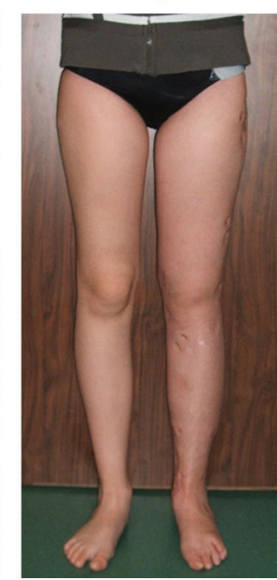

e

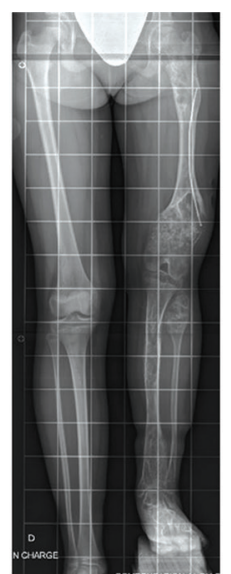

C

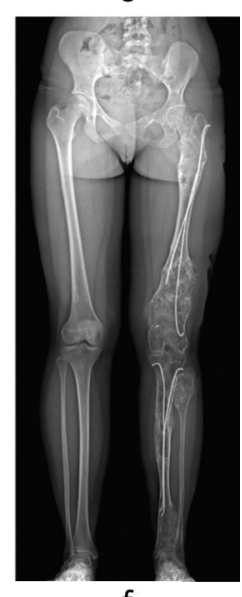

f d

Fig. 7 Maffucci's syndrome in a 14-year old girl. Presence of a small subcutaneous angioma at the basis of the thumb is a sign of Maffucci's syndrome (a). Clinical (b) and radiological aspect (b) before LLD correction. The knee joint is oblique due to a varus of the distal femur and a valgus of the proximal tibia. A double osteotomy of the femur and an osteotomy of the tibia are instrumented with circular external fixators associated with flexible intramedullary nails (d). At the end of the procedure, the knee joint is horizontal and the LLD is corrected (e). The achieved femoral lengthening was then $7.5 \mathrm{~cm}$ and the tibial one was $4.5 \mathrm{~cm}$. The lower limb-healing index was 12.3 days $/ \mathrm{cm}$ (f). Collection of D. Popkov

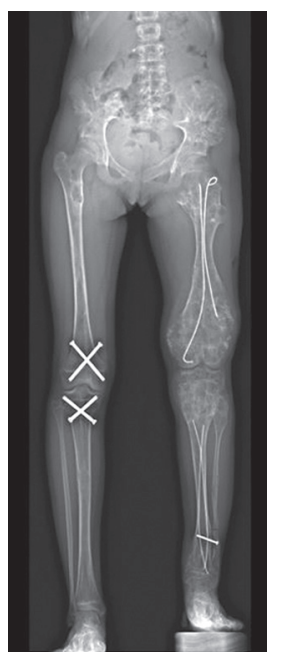

Fig. 8 Ollier's disease with an asymmetric involvement of both lower limbs. The LLD has been treated by a combination of right surgical epiphysis with screws (Métaizeau's technique [18]), and a poly segmental lengthening of the left femur and tibia with an Ilizarov's technique associated with intrameduallary nails (Popkov's technique [17]). Collection P. Journeau

The spine and the ribs may also be involved, as well as the basis of the skull.

\subsection{Clinical signs}

Hands and fingers present with a typic increase of the volume of the bones. Later, finger appear deviated and short. Pathologic fracture may happen as pain is mild. At the forearm, a deformity in varus is frequent, due to the relative shortening of the ulna versus the radius. A progressive dislocation of the radial head leads to an elbow stiffness with flessum and loss of supination. The presence of the dislocated radial head on the lateral and posterior aspect of the elbow demonstrates its progressive migration. The deformity is sometimes similar the deformity observed in the multiple hereditary exostoses (MHE).

Involvement of the lower limbs may induce various deviations as knee valgus and knee varus, and some severe limb leg discrepancies. Femoral neck, legs and feet may also be deviated as well as the whole skeleton of enchondral origin [8].

\subsection{X-rays}

Each location of the disease presents the same aspect than the chondromas described above. The tumor may be of an expansive type, with either a thin cortex or rarely an absence of cortex. Any bone of cartilaginous ossification may be involved, including the skull basis.

Typical lesions are longitudinal lytic columns between the physis and the diaphysis. They present some longitudinal septa in a "W" shape. Asymmetry is almost always observed. Because the mechanism of these bone tumors is a dysregulation of the normal growth, the ossification of the metaphysis is abnormal, and some cartilaginous cells migrate year after year into the metaphysis.

In parallel, X-rays demonstrate the bone deformities, the complex deviations and the limb leg discrepancies (LLD).

\subsection{Pathology}

Enchondromatosis tumors are cartilaginous tumors comparable to chondromas. But they look often more active, with more hyperchromatic chondrocytes and double nuclei. One difficulty is to differentiate them from low grade chondrosarcomas. For Jaffe, some disease should be considered as a pre sarcomatous status [9].

\subsection{Genetics}

Even if the disease is not inherited, some sporadic family cases are described. A dysregulation or some mutations of the gene PTH1R create a disorder of the parathyroid hormone related protein (PTHrP) which regulates the transformation of the hypertrophic zone of the physis, and of the Indian Hedhog ( $\mathrm{IHH})$ which activates the chondrocytes [10].

\subsection{The Maffucci'syndrome}

Described by Maffucci 18 years before Ollier, the bone disorders are strictly similar to those of the Ollier's disease. The main difference is the association with some cutaneous angiomas which should be detected during the clinical evaluation [11]. If the bone evolution is quite similar with the multiple enchondromatosis, some sarcomas may develop in internal organs: ovary, uterus, pancreas.

\subsection{Evolution and prognosis}

Growth disorders are due to the dysregulation of the normal growth. Growth may be slowed down and is asymmetric. Limb discrepancy is usual, increasing with growth, and final bone length remains unpredictable. In addition, deformities like valgus, varus, recurvatum, antecurvatum are also frequent. In forearm, a difference of growth between radius and ulna leads to wrist deviation, and radial head dislocation. 
The bone tumors grow by themselves and their volume increases with time.

The transformation in chondrosarcomas at adulthood seems to be observed in 20 to $30 \%$ of cases, in $50 \%$ of the Maffucci's syndromes. Flat bones are at risk of sarcomas as finger lesions remains benign.

\subsection{Treatment}

The disease has not known treatments of the bone tumors except surgery. It is obviously not possible to treat each lesion. The two goals of treatment are: 1) remove the big tumors responsible of dysfunction, pain, or nerve entrapment; 2) protect the limb function which means to correct bone deformities and limb leg discrepancies.

\subsubsection{Pathologic fractures}

Fractures are reported in almost one third of the cases. They frequently occur in the enchondromatosis areas, but they may appear sometimes in the normal bones. Consolidation is obtained in a normal delay and their treatment is adapted to the site of the fracture and the age of the child.

\subsubsection{Upper limb}

A severe involvement of the proximal humerus may lead to a severe discrepancy at the end of growth. Upon request, bone lengthening may correct the short humerus. Bone union is obtained faster, and complications are less frequent than in congenital diseases.

The most frequent lesion of the forearm is the association of a distal ulnar chondroma responsible of a short ulna to an ulnar deviation of the radius with a progressive loss of pronation and supination. When the progression of the asymmetric growth of both bones reaches $2 \mathrm{~cm}$ or more on the ulna, a progressive dislocation of the radial head appears. Prevention of the radial head dislocation needs frequently an ulnar lengthening and an osteotomy of the radius to strengthen it, sometimes in association with a resection of the ulnar chondroma [12].

The tumors of the hand should be treated before they become enormous in volume. In severe forms, the resection of the tumors may be hemorrhagic, and the thin skin around may become necrotic after extensive surgical approaches. Bone curettage, bone grafting and osteosynthesis are decided accordingly to each case.

\subsubsection{Lower limbs}

The growth disorders of the lower limbs may associate complex 3D bone deformities and LLD. Any type and severity of deviation are observed. The growth dysfunction is responsible of a permanent increase of the troubles, even after a perfect surgical correction, leading to recurrence [13]. As the growth is not predictable, prognosis at the end of growth remains uncertain.

The surgical bone corrections by osteotomy do not present any particular difficulty compare to normal bones, and bone union delay is normal, even when the osteotomy is performed through a chondroma. But before the end of growth, it is mandatory to overcorrect the correction of a value which is difficult to determine.

Around $50 \%$ of the patients present with a LLD. For Shapiro, differences are between 4.3 and $35.7 \mathrm{~cm}$ [14]. Regarding final prevision, it is well-known that:

- The potential of growth decreases with the growth as if the capacity of growth disappears with years.

- The importance of the growth defect is related with the severity of the physeal involvement, but this remains not predictable.

However, our experience of bone lengthening in Ollier's disease allows us to give some comments and recommendations:

- The surgical bone lengthening's in the multiple enchondromatosis do not lead to more complications than in congenital diseases. Indeed, soft tissues grow normally and lengthening itself is quite easy.

- We may anticipate a kind of weakness of the chondromas when they are transfixed by wires and pins. In reality, their biomechanics resistance is good as far as we use circular external fixators with multiple wire fixations.

- The development of the bone regenerate is usually normal, even when it is situated into a chondroma.

- In order to decrease the healing index and to avoid the risk of re fractures after the removal of the external fixator, the addition of two flexible intramedullary nails is helpful as described by Popkov $[15,16]$. Indeed, "a substantially reduced duration of external fixation, limited of postoperative complications and prevention of later pathologic fractures are the reported advantages of the associated use of a circular external fixator with an ESIN system in the management of Ollier's disease" [17] (Fig. 7).

- Surgical epiphysiodesis to correct LLD are rarely indicated as the growth prediction is uncertain. But they may be helpful to reduce the amount of lengthening in severe LLD (Fig. 8).

\section{CONCLUSION}

Chondromas are benign bone tumors which may be responsible of pathologic fractures. Their surgical treatment consists in a curettage and bone grafting or bone-cement filling with or without osteosynthesis. Multiple enchondromatosis should be considered as an osteochondrodysplasia. Its treatment is not the treatment of the multiple chondromas themselves, but of the bone deformities and LLD induced by the disorder. The transformation of some tumours in chondrosarcomas in adolescence or adulthood needs a strict follow-up of these patients.

\section{REFERENCES}

1. Mazabraud A. Anatomie pathologique osseuse tumorale: Experience personnelle [Tumor Bone Pathology Anatomy: Personal Experience]. Paris, Springer-Verlag, 1994, 552 p. (in French)

2. M. Campanacci, Bertoni F., Bacchini P., Enneking W.F. Bone and soft tissue tumors. Translated by Notini S. New-York, Springer-Verlag, 1990, $1131 \mathrm{p}$.

3. Lichtenstein L., Hall J.E. Periosteal chondroma: a distinctive benign cartilage tumor. J. Bone Joint Surg. Am., 1952 , vol. 24 A, no. 3, pp. $691-697$.

4. Jesus-Garcia R., Osawa A., Filippi R.Z., Viola D.C.M., Korukian M., De Carvalho Campos Neto G., Wagner J. Is PET-CT an accurate method for the differential diagnosis between chondroma and chondrosarcoma? SpringerPlus, 2016, vol. 5, article No 236. DOI: 10.1186/s40064-016-1782-8.

5. Deckers C., Schreuder B.H., Hannink G., de Rooy J.W., van der Geest I.C. Radiologic Follow-Up of Untreated Enchondroma and Atypical Cartilaginous Tumors in the Long Bones. J. Surg. Oncol., 2016, vol. 114, no. 8, pp. 987-991. DOI: 10.1002/jso.24465. 
6. Omlor G.W., Lohnherr V., Lange J., Gantz S., Mechtersheimer G., Merle C., Raiss P., Fellenberg J., Lehner B. Outcome of conservative and surgical treatment of enchondromas and atypical cartilaginous tumors of the long bones: retrospective analysis of 228 patients. BMC Musculoskelet. Disord., 2019, vol. 20, no. 1, pp. 134. DOI: 10.1186/s12891-019-2502-7.

7. Ollier L. De la dyschondroplasie [Dyschondroplasia]. Bull. Soc. Chir. (Lyon), 1899, vol. 3, pp. 22-27. (in French)

8. Kumar A., Jain V.K., Bharadwaj M., Arya R.K. Ollier Disease: Pathogenesis, Diagnosis, and Management. Orthopedics, 2015, vol. 38, no. 6, pp. e497-e506. DOI: 10.3928/01477447-20150603-58.

9. Jaffe H.L. Tumors and tumorous conditions of the bones and joints. Philadelphia, Pa. Lea \& Febiger, 1958,629 p.

10. Couvineau A., Wouters V., Bertrand G., Rouyer C., Gérard B., Boon L.M., Grandchamp B., Vikkula M., Silve C. PTHR1 mutations associated with Ollier disease result in receptor loss of function. Hum. Mol. Genet., 2008, vol. 17, no. 18, pp. 2766-2775. DOI: 10.1093/hmg/ddn176.

11. Maffucci A. Di un casa di encondroma ed angioma multiplo. Contribuzione alia genesi embrionale dei tumori [Of a case of enchondroma and multiple angioma; contribution to the embryonic genesis of tumors]. II Movemento Med. Chir. Napoli, 1881, vol. 3, pp. 399-412. (in Italian)

12. Finidori G., Rigault P., Padovani J.P. Anomalies de croissance du squelette anti-brachial dans la maladie exostosante et dans la maladie d'Ollier [Growth disturbance of the forearm in multiple exostosis and dyschondroplasia (author's transl)]. Chir. Pediatr., 1979, vol. 20, no. 2, pp. 143-148. (in French)

13. Reif T.J., Matthias J., Fragomen A.T., Rozbruch S.R. Limb Length Discrepancy and Angular Deformity due to Benign Bone Tumors and Tumor-like Lesions. Am. Acad. Orthop. Surg. Glob. Res. Rev., 2021, vol. 5, no. 3, pp. e00214. DOI: 10.5435/JAAOSGlobal-D-20-00214.

14. Shapiro F. Ollier's disease. An assessment of angular deformity, shortening, and pathological fracture in twenty-one patients. J. Bone Joint Surg. Am., 1982, vol. 64, no. 1, pp. 95-103.

15. Popkov D., Popkov A., Haumont T., Journeau P., Lascombes P. Flexible Intramedullary Nail Use in Limb Lengthening. J. Pediatr Orthop., 2010, vol. 30, no. 8, pp. 910-918. DOI: 10.1097/BPO.0b013e3181f0eaf9.

16. Jager T., Popkov D., Lascombes P., Popkov A., Journeau P. Elastic intramedullary nailing as a complement to Ilizarov’s method for forearm lengthening: a comparative pediatric prospective study. Orthop. Traumatol. Surg. Res., 2012, vol. 98, no. 4, pp. 376-382. DOI: 10.1016/j. otsr.2012.01.007.

17. Popkov D., Journeau P., Popkov A., Haumont T., Lascombes P. Ollier's disease limb lengthening: should intramedullary nailing be combined with circular external fixation? Orthop. Traumatol. Surg. Res., 2010, vol. 96, no. 4, pp. 348-353. DOI: 10.1016/j.otsr.2010.01.002.

18. Métaizeau J.P., Wong-Chung J., Bertrand H., Pasquier P. Percutaneous epiphysiodesis using transphyseal screws (PETS). J. Pediatr. Orthop., 1998, vol. 18, no. 3, pp. 363-369.

Received: 12.05.2021

\section{Information about the authors:}

1. Pierre Lascombes, Honorary Professor University of Nancy, France; Past Professor, University of Geneva, Switzerland, Email: pierrelascombes@gmail.com

2. Laurence Mainard-Simard,

Paediatric Radiology, Hôpital d'Enfants, CHU de Nancy, Vandoeuvre les Nancy, France 